\title{
Criterios de Evaluación Odontológica Pre-Radioterapia y Necesidad de Tratamiento de las Enfermedades Orales Post-Radioterapia en Cabeza y Cuello
}

\author{
Pre-Radiotherapy Dental Evaluation Criteria and Treatment Needs \\ of Oral Side Effects after Head and Neck Radiotherapy
}

\author{
Wilfredo Alejandro González-Arriagada*; Alan Roger Santos-Silva*; \\ Marco Aurélio Carvalho de Andrade*; Rogério de Andrade Elias ${ }^{* *}$ \& Marcio Ajudarte Lopes ${ }^{* *}$
}

GONZÁLEZ-ARRIAGADA, W. A.; SANTOS-SILVA, A. R.; CARVALHO DE ANDRADE M. A.; ELIAS, R. A. \& LOPES, M. A. Criterios de evaluación odontológica pre-radioterapia y necesidad de tratamiento de las enfermedades orales post-radioterapia en cabeza y cuello. Int. J. Odontostomat., 4(3):255-266, 2010.

RESUMEN: El objetivo de esta revisión es presentar criterios de evaluación odontológica pre-radioterapia y cuáles son las principales necesidades de tratamiento de estos pacientes después del tratamiento de radiación. Fueron revisados artículos en inglés, español y portugués entre 1995 y 2009 indexados en Pubmed y Scielo. Las palabras claves fueron: cáncer oral y radioterapia, complicaciones de la radioterapia en cabeza y cuello, evaluación oral pre-radioterapia.Los efectos colaterales de la radioterapia en la región de cabeza y cuello pueden ser temporales o tardíos. Aquellos que son tardíos, como la caries de radiación y la osteorradionecrosis pueden estar directamente relacionados con el hecho de no haber realizado una evaluación odontológica previa y pueden afectar severamente la calidad de vida del paciente.La participación del cirujano-dentista en el equipo multidisciplinario y la evaluación odontológica de los pacientes que serán sometidos a radioterapia en la región de cabeza y cuello son de vital importancia en mejorar la calidad de vida post-operatoria de estos pacientes.

PALABRAS CLAVE: radioterapia, cáncer de cabeza y cuello, efectos colaterales, necesidades de tratamiento.

\section{INTRODUCCIÓN}

La literatura en las últimas décadas ha resaltado la importancia de la evaluación odontológica preradioterapia en el mejoramiento de la calidad de vida de los pacientes con cáncer oral, disminuyendo las necesidades de tratamiento odontológico restaurador o quirúrgico post-radioterapia. Además, se ha enfatizado en la importancia de la participación del cirujano-dentista en el equipo multidisciplinario responsable por la salud del paciente oncológico (Specht, 2002; Schiødt \& Hermund, 2002; Jham \& Silva Freire, 2006; Miller \& Quinn, 2006; Rosales et al., 2009).

El cáncer oral corresponde aproximadamente al $3 \%$ de los casos de cáncer en el ser humano
(Greenlee et al., 2000), siendo el carcinoma espinocelular (CEC), el tumor maligno más frecuente de la cavidad oral, con aproximadamente un $90 \%$ de los casos de cáncer oral (Ochsenius et al., 2003). El diagnóstico de cáncer oral la mayoría de las veces es en estadios TNM avanzados, por lo que el tratamiento incluye radioterapia y/o quimioterapia, con o sin cirugía previa (Caribé Gomes et al., 2003; Rosales et al.).

La radioterapia afecta tanto a las células malignas como a las células normales que tienen un alto nivel de replicación, produciendo efectos colaterales. Pueden ser clasificados en temporales aquellos que ocurren durante la radioterapia (radiodermatitis,

\footnotetext{
* Cátedra de Diagnóstico y Patología Oral, Escuela de Odontología, Universidad de Valparaíso, Valparaíso, Chile.

* Departmento de Diagnóstico Oral, Semiología y Patología Oral, Escuela Dental de Piracicaba, Universidad Estadual de Campinas (UNICAMP), Piracicaba, São Paulo, Brasil.
} 
GONZÁLEZ-ARRIAGADA, W. A.; SANTOS-SILVA, A. R.; CARVALHO DE ANDRADE M. A.; ELIAS, R. A. \& LOPES, M. A. Criterios de evaluación odontológica pre-radioterapia y necesidad de tratamiento de las enfermedades orales post-radioterapia en cabeza y cuello. Int. J. Odontostomat., 4(3):255-266, 2010.

mucositis, xerostomía, pérdida del gusto y candidosis) o permanentes aquellos que afectan a los pacientes más allá del tratamiento de radiación (caries de radiación y osteorradionecrosis) (Öhrn et al., 2001; Specht; Caribé Gomes et al.; Sciubba \& Goldenberg, 2006; Jham et al., 2008; Rosales et al.).

La participación de cirujano-dentista es importante antes, durante y después de la radioterapia, sobre todo enfocado en la prevención de aquellas complicaciones tardías que pueden tener consecuencias severas para la calidad de vida del paciente. El objetivo de esta revisión es la evaluación de artículos que abordan las necesidades de tratamiento odontológico de los pacientes con cáncer oral después de la radioterapia, con y sin evaluación previa, determinando la eficacia de aquella evaluación, presentando criterios de evaluación odontológica pre-radioterapia.

\section{MATERIAL Y MÉTODO}

Fueron analizados estudios publicados en la literatura médica y odontológica, luego de una revisión sistemática en Pubmed (literatura en inglés) y Scielo (portugués y español). Las palabras claves utilizadas fueron: cáncer oral y radioterapia, complicaciones de la radioterapia en cabeza y cuello, evaluación oral preradioterapia.

Fueron incluidas revisiones sistemáticas, estudios prospectivos longitudinales y estudios retrospectivos, que fueron publicados entre los años 1995 y 2009. Se excluyeron los artículos sin resumen disponible y los casos clínicos.

\section{EFECTOS COLATERALES DE LA RADIOTERAPIA}

En general, los pacientes con cáncer de cabeza y cuello son sometidos a altas dosis de radiación, involucrando cavidad oral, maxilar, mandíbula y glándulas salivares (Jham \& Silva Freire, 2006; Rubira et al., 2006). La radioterapia produce diversos efectos colaterales en las áreas involucradas, la mayoría de ellos dependientes de la dosis de radiación y duración de la terapia, sin embargo, factores propios de los pacientes también deben ser considerados, como mala higiene oral, condición de los tejidos orales, tabaquis- mo y alcohol, sistema inmunitario y rigurosidad en consultas con el cirujano-dentista. Si estos efectos llegan a ser muy discapacitantes para los pacientes, pueden llevar a suspender el tratamiento, incrementando la duración de la terapia y los costos del tratamiento (Specht; Vissink et al., 2003; Rubira et al., 2007; Rosales et al.).

Los efectos colaterales pueden ser clasificados como temporales o agudos, y tardíos o permanentes (Tabla I). Las reacciones temporales ocurren durante el tratamiento y son generalmente reversibles. Las complicaciones tardías llegan a ser irreversibles, disminuyendo considerablemente la calidad de vida del paciente (Jham \& Silva Freire).

\begin{tabular}{l} 
Tabla I. Efectos colaterales de la radio- \\
terapia de cabeza y cuello. \\
\hline Efectos temporales o agudos \\
\hline Mucositis oral \\
Candidosis \\
Disgeusia/Ageusia \\
Dermatitis \\
Trismus \\
Xerostomía/hiposalivación \\
Disfagia \\
\hline Efectos permanentes o tardíos \\
\hline Xerostomía/hiposalivación \\
Disfagia \\
Caries de radiación \\
Osteorradionecrosis \\
\hline
\end{tabular}

La mucositis es la complicación más importante durante el tratamiento y que en casos severos lleva a suspenderlo. La radiación afecta las mucosas orales en su histomorfología, disminuyendo su función de barrera contra infecciones, siendo más susceptible a ser dañada (Handschel et al., 2001). Ésta se desarrolla, generalmente, después de la segunda semana de tratamiento y causa dolor severo, dificultad para tragar, comer y hablar, afectando en gran medida la calidad de vida del paciente (Lopes et al., 1998; Sonis, 2004; Duncan et al., 2005, Sciubba \& Goldenberg; Keefe et al., 2007; Russo et al., 2008; Madan et al., 2008; Rosenthal \& Trotti, 2009). Es más común en mucosa no queratinizada de piso de boca, lengua, mucosa yugal, labios y paladar blando, (Lopes et al.) y aparece en más del $90 \%$ de los pacientes irradiados en cabeza y cuello (Herrstedt et al., 2000; Grötz et al., 2001; Trotti et al., 2003). Puede persistir por dos a tres 

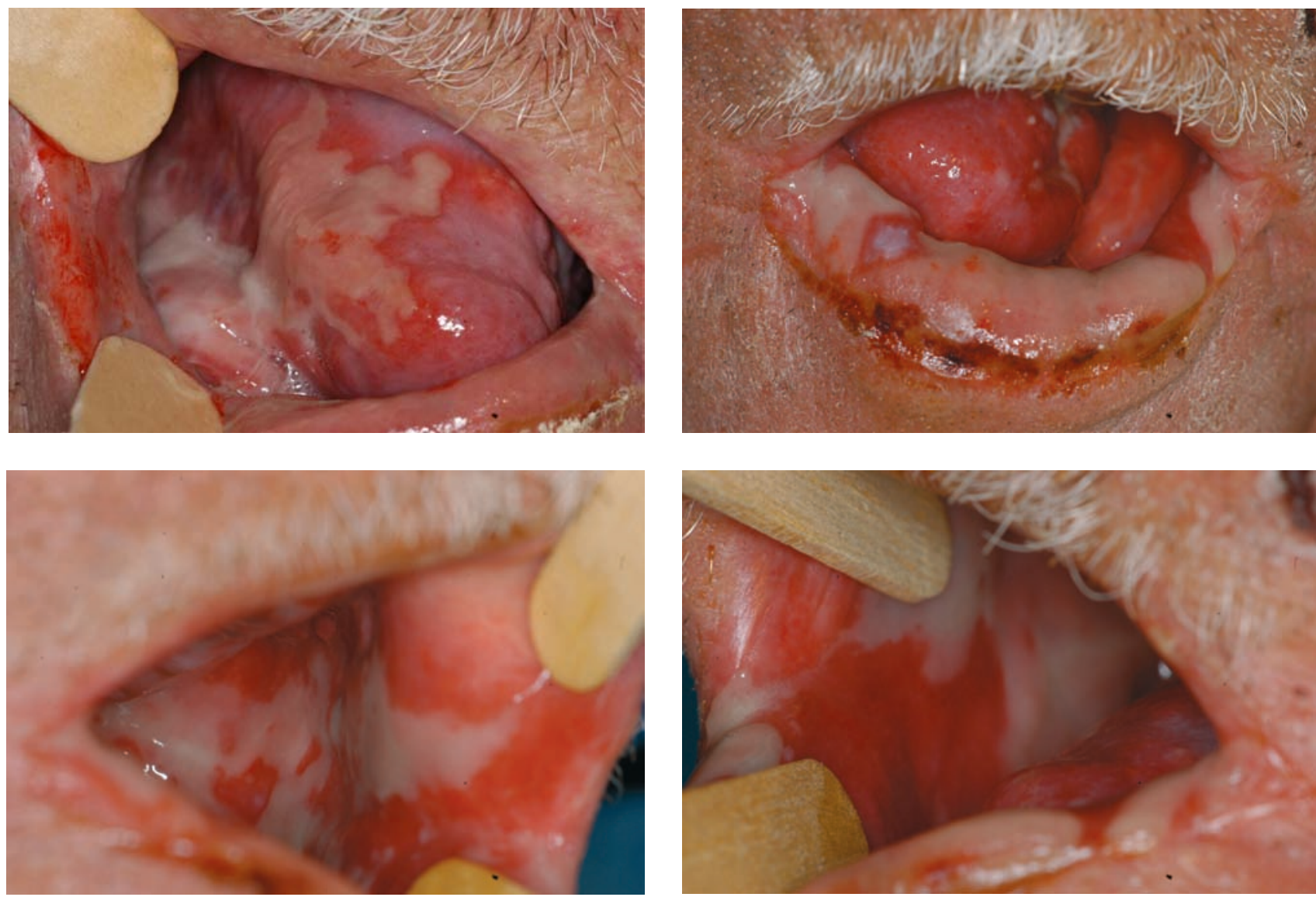

Figs. 1-4. Mucositis oral severa en paciente sometido a tratamiento de radioterapia e quimioterapia por cáncer de lengua en estadio IV, afectando lengua, piso de boca, labios y mucosa bucal bilateral.

semanas después del término de la radioterapia (Köstler et al., 2001; Rosales et al.). La severidad ha sido asociada con la dosis y duración de la terapia, edad, condición nutricional, microbiota oral, nivel de higiene bucal, secreción salival y uso de tabaco, (Trotti et al.; Scully et al.; Shih et al.) y puede ser más severa cuando la radioterapia es asociada con quimioterapia (Öhrn et al., 2001; Scully et al., 2003; Jham \& Silva Freire) (Figs. 1-4).

La evolución de la mucositis ocurre en cuatro etapas: inflamatoria/vascular, epitelial, ulcerativa/ bacteriológica y de cicatrización. (Shih et al.; Scully et al., 2003) La escala más usada para medir mucositis oral es de la OMS, que la clasifica en: grado 0 , cuando no hay signos ni síntomas; grado 1 , cuando la mucosa está eritematosa y dolorosa; grado 2, caracterizado por úlceras, el paciente puede comer normalmente; grado 3, cuando el paciente tiene úlceras y sólo puede beber líquidos; y grado 4, cuando el paciente no puede comer ni beber (Scully et al., 2004; Jham \& Silva Freire). Las principales complicaciones relaciona- das con la mucositis asociada a radioterapia son dolor bucal, infección, disgeusia, pérdida de peso y malnutrición (Shih et al.).

La candidosis es la infección más común de la cavidad oral de los pacientes que reciben radioterapia, y afecta generalmente paladar blando, lengua y mucosa yugal (Ramirez-Amador et al., 1997; Redding et al., 1999; Köstler et al.; Grötz et al., 2003; Miller \& Quinn; Corvò et al., 2008) (Figura 5). Generalmente es de tipo pseudomembranosa, caracterizada por la formación de placas blancas, removibles al raspado, aunque se puede presentar de tipo eritematosa en algunos casos (Lopes et al.; Jham \& Silva Freire). Los principales factores de riesgo en pacientes irradiados para desarrollar candidosis son xerostomía, uso prótesis dental y tabaquismo (Redding et al.; Nicolatou-Galitis et al., 2003; Sciubba \& Goldenberg; Corvò et al.).

La radiodermatitis afecta la piel involucrada dentro del campo de radiación, presentando alteraciones como eritema, formación de bulas, descamación, 
necrosis, ardor y dolor (Fig. 6). La regresión ocurre poco después del término de la radioterapia (Lopes et al.).

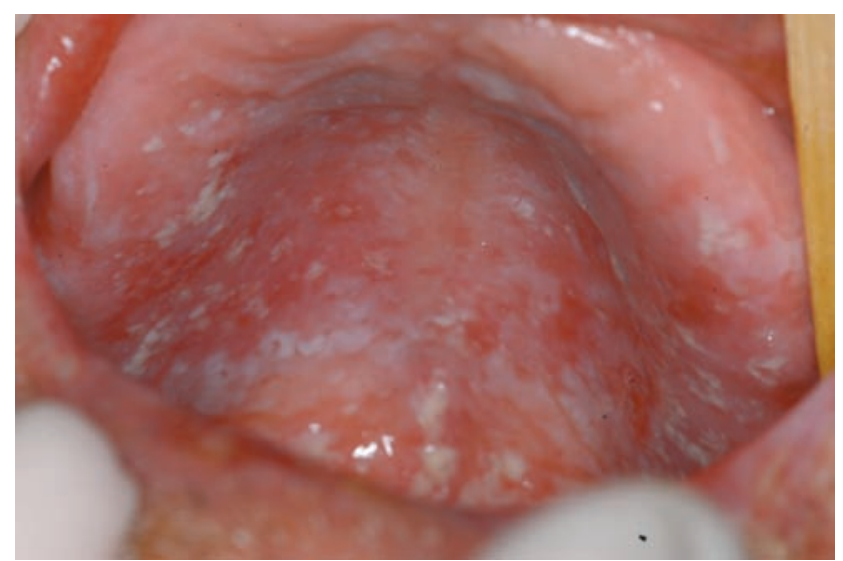

Fig. 5. Candidosis en paciente sometido a radioterapia por cáncer de lengua en estadio IV afectando reborde alveolar superior, paladar duro y blando.

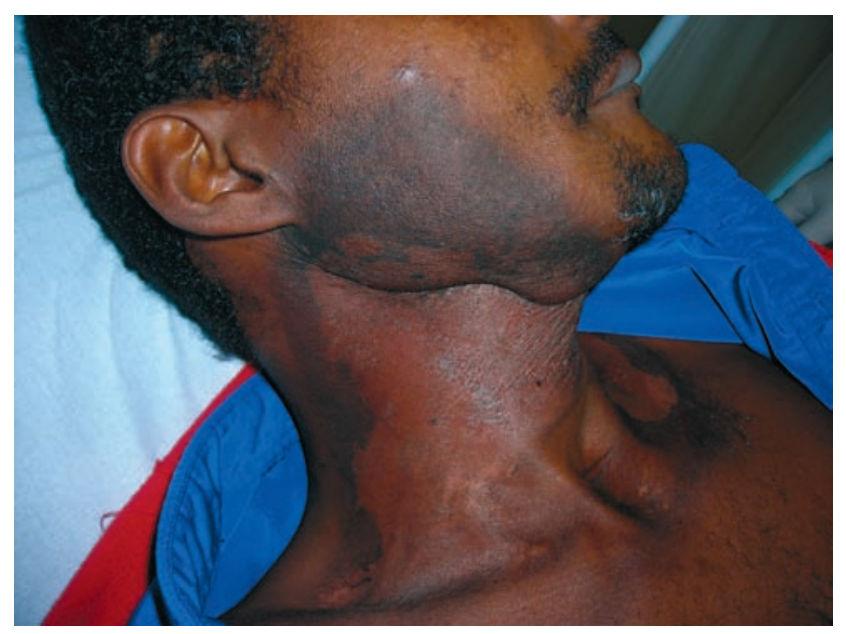

Fig. 6. Paciente sometido a radioterapia asociada a cirugía por carcinoma espinocelular de orofaringe con dermatitis en área expuesta a radiación, unos días después de finalizado el tratamiento.

La dosis acumulativa de radiación puede provocar disgeusia y pérdida del gusto, transitoria en la mayoría de los pacientes sometidos a radioterapia, desde la segunda o tercera semana de terapia (Jham \& Silva Freire). La pérdida del gusto ocurre como un efecto directo de la radiación en las papilas gustativas y a cambios en la saliva, retornando después de la terapia (Vissink et al.). Ha sido relatada la presencia de disestesia en pacientes irradiados por cáncer de lengua (Sharp et al., 2008).

La disfagia es otra complicación de la radioterapia, asociada con xerostomía y tipo de cirugía. Por otro lado, se han reportado problemas de habla y deglu- ción, severos y persistentes, en pacientes con tratamiento de radioterapia asociada con remoción quirúrgica del tumor en tumores en estadios avanzados y localizados en lengua y piso de boca (SuarezCunqueiro et al., 2008).

Puede acontecer trismus cuando la articulación temporomandibular y los músculos masticadores están incluidos en los campos de radiación, limitando la apertura bucal (Hancock et al., 2003; Vissink et al.). Generalmente es temporal, pero sin tratamiento puede ser permanente (Sciubba \& Goldenberg). Es provocado por fibrosis de los músculos pterigoídeos, articulaciones temporomandibulares y tejidos blandos adyacentes (Chua et al., 2001).

Pueden observarse también alteraciones funcionales de las glándulas salivares como disminución del flujo salivar, modificación en la composición de saliva (concentración de electrolitos, inmunoglobulinas, $\mathrm{pH}$ ) y sobrepoblación de microflora criogénica y fúngica, dentro de las primeras semanas de tratamiento. (Niedermeier et al., 1998; Grötz et al., 2001; Reddy et al., 2002; Bonan et al., 2003; Malouf et al., 2003, Jensen et al., 2003; Dirix et al., 2006; Chambers et al., 2008; Chitra \& Shyamala Devi, 2008) Estas alteraciones pueden ser permanentes por el daño de la radiación a la membrana plasmática y DNA de las células acinares, disminuyendo su capacidad secretora (Konings et al., 2005). Corresponde a una alteración dosis-dependiente, y los acinos serosos son más sensibles que los mucosos (Garg \& Malo, 1997).

La xerostomía es una de las complicaciones más frecuentes, que acarrea otras complicaciones adicionales, afectando en gran medida la calidad de vida del paciente (Guchelaar et al., 1997; Ramirez Amador et al.; Vissink et al.; Grötz et al., 2003; Jensen et al.; Pow et al., 2006; Miller \& Quinn; Rubira et al., Dirix et al.) (Fig. 7). Puede facilitar el desarrollo de mucositis, caries de radiación, enfermedades periodontales, infecciones fúngicas, bacterianas y virales (Guchelaar et al.; Ramirez-Amador et al.; Garg \& Malo; Niedermeier et al.; Epstein et al., 2002; Toljanic et al., 2002; Pow et al., 2003; Sonis et al.; Sciubba \& Goldenberg; Chitra \& Shyamala Devi) El $80 \%$ de los pacientes irradiados pueden sufrir de xerostomía, por lo que es importante evaluar la percepción individual y la verdadera disminución del flujo salivar durante el seguimiento de estos pacientes (Jham \& Silva Freire). La función de las glándulas se recupera generalmente de seis a doce meses después del término de la radioterapia y el retorno del flujo salivar normal depende de la dosis total de radia- 


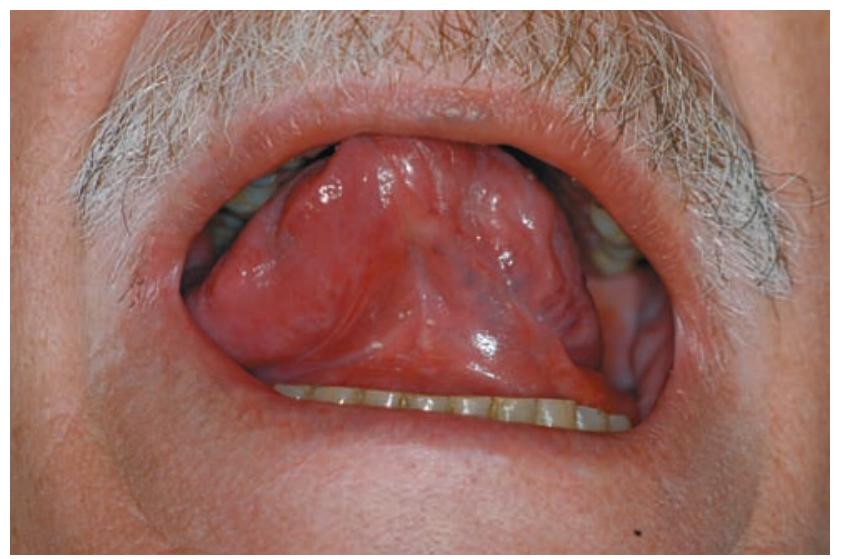

Fig. 7. Paciente sometido a radioterapia por cáncer en base de lengua, relatando xerostomía severa. En la foto se puede apreciar la hiposalivación, causando sequedad bucal.

ción y de la cantidad de glándulas salivares localizadas en el campo irradiado, aunque en algunos casos puede no volver a niveles normales de flujo salivar (Lopes et al.; Jensen et al.; Rubira et al.).

La caries de radiación es una de las complicaciones tardías de la radioterapia, debido a efectos directos $\mathrm{y}$, principalmente indirectos de la radiación sobre los dientes, siendo la hiposalivación el más importante (Vissink et al.; Kielbassa et al.). Comparado con las caries no asociadas a radioterapia, el padrón clínico es distinto y de progresión rápida, siendo las superficies lisas afectadas primeramente, desarrollando lesiones radiculares e incisales en forma de cavidades y/o manchas cafés (Silva et al., 2009) (Fig. 8). La literatura ha descrito que la respuesta pulpar es alterada por la radiación, presentando una reducción de la función de los odontoblastos (Kielbassa et al., 1995), sin embargo, se ha relatado la presencia de zona translúcida, dentina reaccional e intratubular en dientes extraídos de pacientes irradiados, mostrando que el padrón morfológico y de desmineralización frente a la caries es el mismo que en pacientes no irradiados, demostrando la mayor importancia de los efectos indirectos (Silva et al.).

La osteorradionecrosis es una complicación tardía seria, asociada a altas dosis de radiación y tiene una incidencia entre 2 y $22 \%$ (Jereczek-Fossa \& Orecchia, 2002; Bonan et al.; Sciubba \& Goldenberg). La radiación provoca cambios irreversibles en el hueso por estrechamiento de los canales vasculares, llevando a la disminución del flujo sanguíneo en la área afectada, pérdida de osteocitos y osteoblastos, reducción de formación de osteoide y fibrosis en la médula

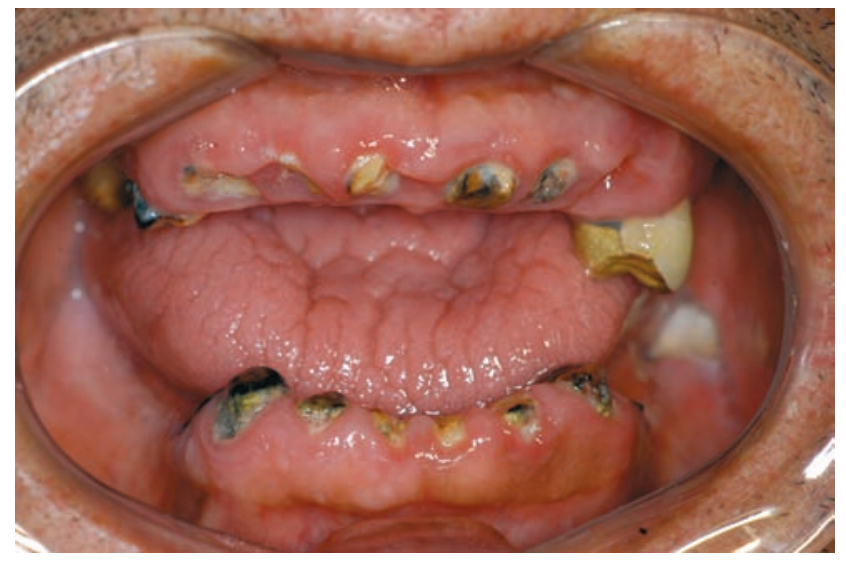

Fig. 8. Paciente sometido a radioterapia, sin evaluación odontológica previa ni seguimiento odontológico periódico, un año y medio después del tratamiento. Observar agresividad de la caries y prácticamente destrucción total de las piezas dentarias.

ósea. La menor irrigación causa mayor vulnerabilidad a la infección y necrosis. (Scully \& Epstein, 1996; Wong et al., 1997; Thorn et al., 2000; Jereczek-Fossa \& Orecchia; Hancock et al.; Miller \& Quinn). La osteorradionecrosis puede ocurrir espontáneamente o más comúnmente, después de un trauma (generalmente exodoncias). Es más común en hombres, y la mandíbula es más afectada que el maxilar, (Reuther et al., 2003; Jham \& Silva Freire) principalmente en el área de los molares inferiores (82\%) (Thorn et al.) (Fig. 9).

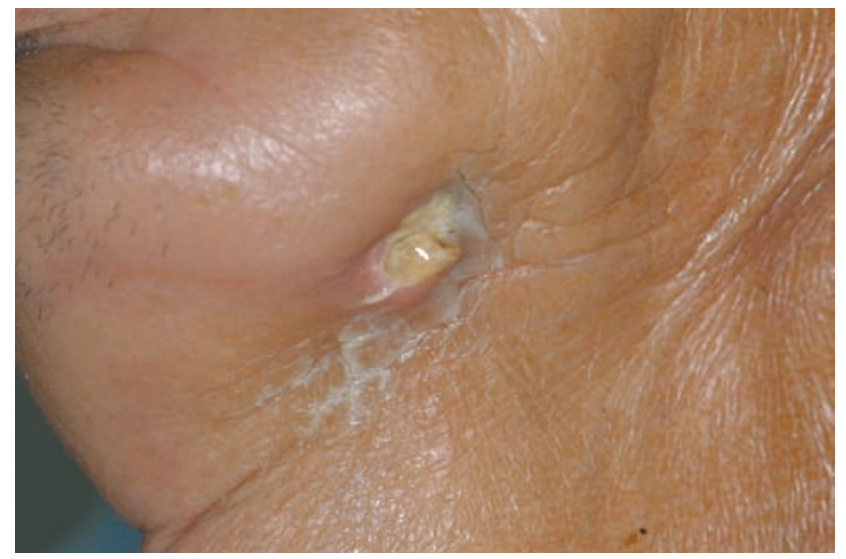

Fig. 9. Fístula provocada por ostorradionecrosis de mandíbula un año después de concluida la radioterapia. El paciente presentaba una pésima condición oral y trismus, además no fue realizado ningún tratamiento odontológico preventivo antes de la radioterapia

Uno de los principales factores de riesgo de osteorradionecrosis es la exodoncia post-radioterapia y el riesgo persiste por toda la vida del paciente (Thorn et al., 2000; Oh et al., 2004; Koga et al.). Se aconseja 
evitar extracciones post-radioterapia, por lo que deben ser realizadas por lo menos dos semanas antes del inicio de la radioterapia (Bonan et al.; Rosales et al.). Otros factores asociados a osteorradionecrosis son tumores avanzados, resección mandibular, uso de alcohol y tabaco, pobre estado nutricional y mala higiene oral (Andrade et al., 2003; Reuther et al., 2003). Esta complicación generalmente está asociada a edema, sobreinfección (fistulación y celulitis) y fracturas patológicas, en $15 \%$ de los pacientes, siempre acompañados de dolor, que en la mayoría de los casos cede a analgésicos (Wong et al.; Németh et al., 2000, Sciubba \& Goldenberg; Jham \& Silva Freire).

\section{CRITERIOS DE EVALUACIÓN DEL ESTADO ORAL DE LOS PACIENTES PRE-RADIOTERAPIA}

La morbilidad de las complicaciones orales de la radioterapia es una razón importante para que un cirujano-dentista esté envuelto en el tratamiento del paciente oncológico antes, durante y después de que sean irradiados (Specht; Hancock et al.). Han sido reportados estudios con pacientes examinados antes de la radioterapia que muestran que el $82 \%$ de los pacientes tienen necesidad de exodoncias y el $93 \%$ de ellos tienen enfermedad periodontal moderada a severa. Las exodoncias y el tratamiento periodontal previo a la radioterapia previenen el desarrollo de caries de radiación, progresión de enfermedad periodontal y osteorradionecrosis (Scully \& Epstein; Epstein et al.; Bonan et al.; Kielbasssa et al.; Jham et al.).
El manejo odontológico de pacientes que serán sometidos a radioterapia debe considerar: 1) identificar enfermedades dentales existentes y riesgos potenciales de enfermedades; 2) eliminar focos dentales/orales infecciosos antes de iniciar la radioterapia; 3) informar a los pacientes de efectos colaterales esperados; 4) establecer un sistema adecuado de higiene oral para incrementar el éxito; 5) proveer un plan de mantención de higiene oral y tratamiento con flúor, para rehabilitación y tratamiento; 6) establecer la necesidad de colaboración multidisciplinaria en el tratamiento de secuelas y síntomas orales, durante y después de la radioterapia (Epstein \& Scully; Garg \& Malo; Andrews \& Griffiths, 2001; Schiødt \& Hermund; Toljanic et al.; Hancock et al.; Kielbasssa et al.; Miller \& Quinn; Papas et al., 2008) (Fig. 10). Todos los dientes con pronóstico cuestionable deben ser extraídos antes de la radioterapia, a fin de evitar cirugías después del tratamiento, dejando un tiempo adecuado para cicatrización (Andrews \& Griffith; Vissink et al.) (Tabla II).

El pronóstico del tumor debe ser considerado como prioritario en la decisión del tratamiento (Andrews \& Griffiths). Si el tratamiento radioterápico es paliativo, sólo deben ser eliminados los focos de infección, visto que los efectos colaterales no ocurrirán debido a la menor sobrevida del paciente (Andrews \& Griffiths; Rosales et al.). Los protocolos de atendimiento odontológico pre y post-radioterapia son realizados con la finalidad de limitar los riesgos provenientes de esta terapia (Rosales et al.). Los mejores resultados en el manejo de las complicaciones de la radioterapia se obtienen cuando se incluye prevención y seguimiento periódico (Schiødt \& Hermund; Miller \& Quinn).

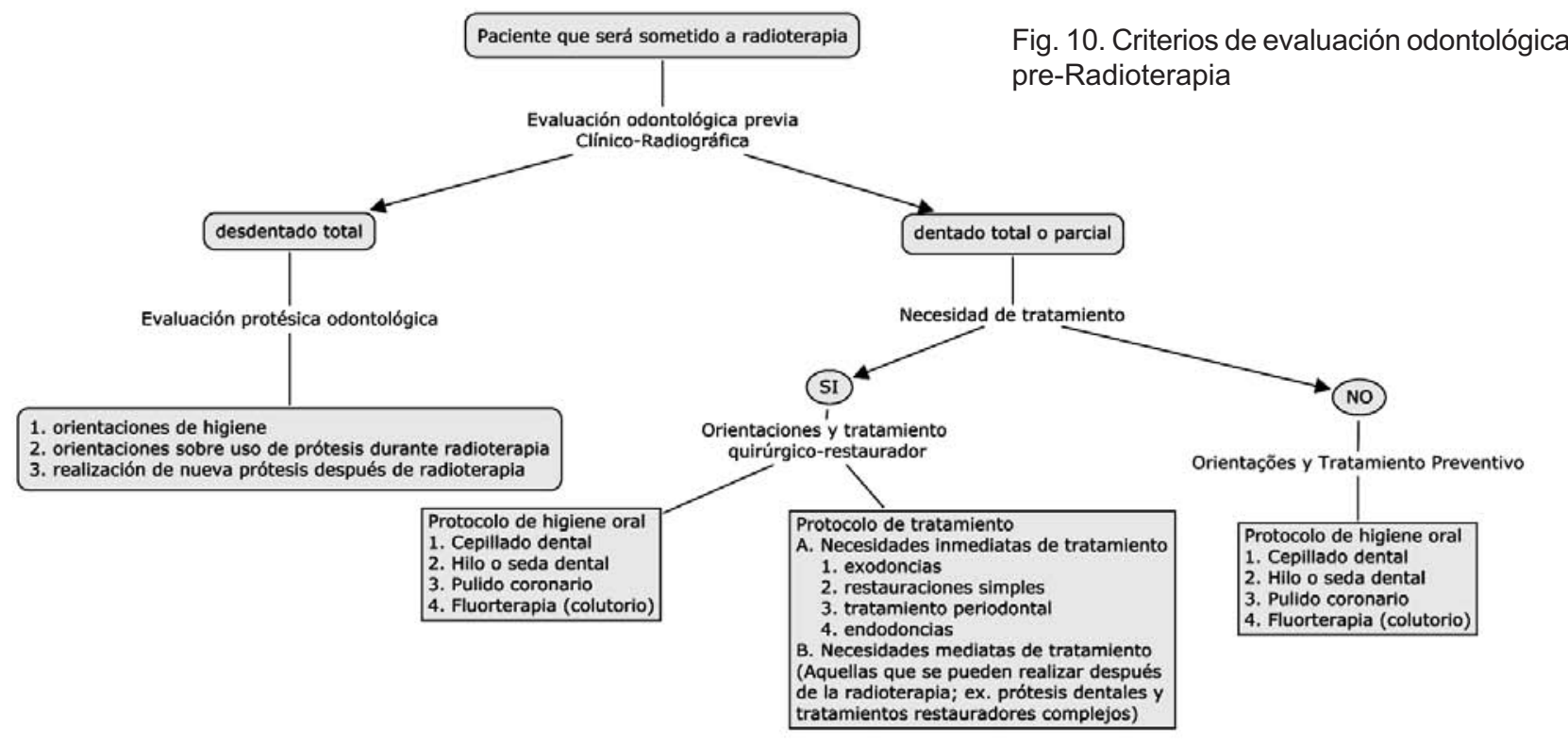


Tabla II. Criterios para exodoncia de dientes pre-radioterapia.

\section{¿En qué casos deben ser extraídos los dientes pre-radioterapia?}

1. Caries (que no es posible restaurar)

2. Periodontitis apical sintomática

3. Enfermedad periodontal moderada a severa

4. Diente impactado o semierupcionado

5. Lesión periapical extensa (crónica)

6. Indicación protésica (ex. diente sobreerupcionado, ausencia de antagonista, diente mesializado)

Observación 1: en casos que el paciente tenga una higiene oral deficiente o esté discapacitado para realizar su higiene dental, es prefe rible realizar la exodoncia de todos los dientes y planificar la rehabilitación con prótesis totales.

Observación 2: si es posible, es preferible optar por el tratamiento de endodoncia y luego amputación coronaria en lugar de la realización de una exodoncia.

Observación 3: en caso de enfermedad periodontal severa asintomática, se recomienda no realizar la exodoncia y esperar la exfoliación espontánea del diente involucrado.

\section{NECESIDAD DE TRATAMIENTO ODONTOLÓGICO POST-RADIOTERAPIA}

El rango de edad y género de los pacientes incluidos en los estudios evaluados son similares, principalmente mayores de 40 años de edad y hombres (Ramirez-Amador et al.; Doerr \& Marunick, 1997; Handschel et al.; Toljanic et al., 2002; Koc \& Aktas, 2003; Katz et al., 2004; Oh et al.; Rosales et al., 2009; Bonan et al.; Jham et al.; Papas et al., 2008; SuarezCunqueiro et al.). La fuerte relación de consumo de tabaco y bebida alcohólica con ocurrencia de cáncer hace estos factores importantes de considerar en la evaluación pre-terapéutica (Rosales et al.). El perfil típico de los pacientes con cáncer de cabeza y cue-

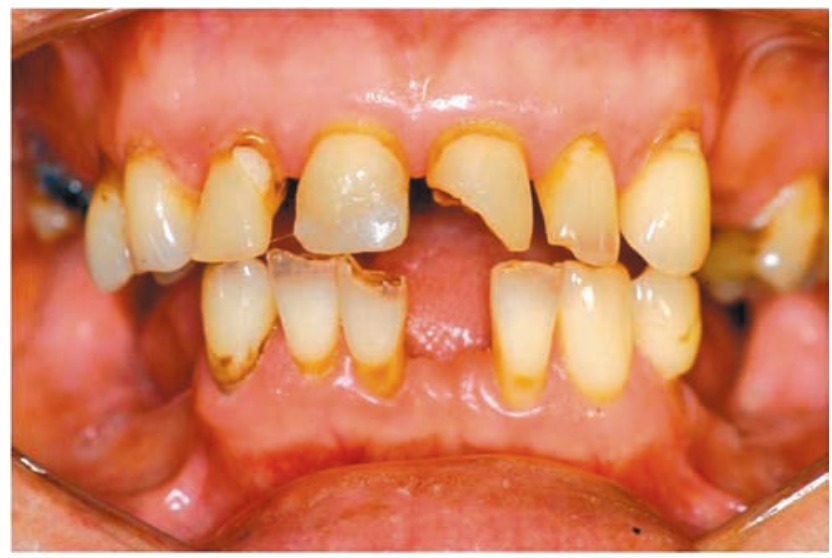

Fig.11. paciente sometida a radioterapia con caries de radiación en varios dientes, ausencias dentarias y restauraciones en mal estado.
Ilo, además incluye un bajo nivel socioeconómico y una pobre condición dental (Specht; Bonan et al., 2006).

La mayoría de las necesidades post-radioterapia de los pacientes evaluados corresponde a confección de prótesis removibles. Las prótesis son realizadas después del término del tratamiento (Rosales et al.). Los pacientes desdentados deben ser instruidos a no usar las prótesis durante la terapia, y hasta dos meses después del término del tratamiento, nuevas prótesis deben ser confeccionadas (Lopes et al.). Los pacientes que tienen dientes en buenas condiciones, pueden mantenerlos y son orientados a cuidados odontológicos post-radioterápicos junto a profesionales con experiencia en esta área. Además,

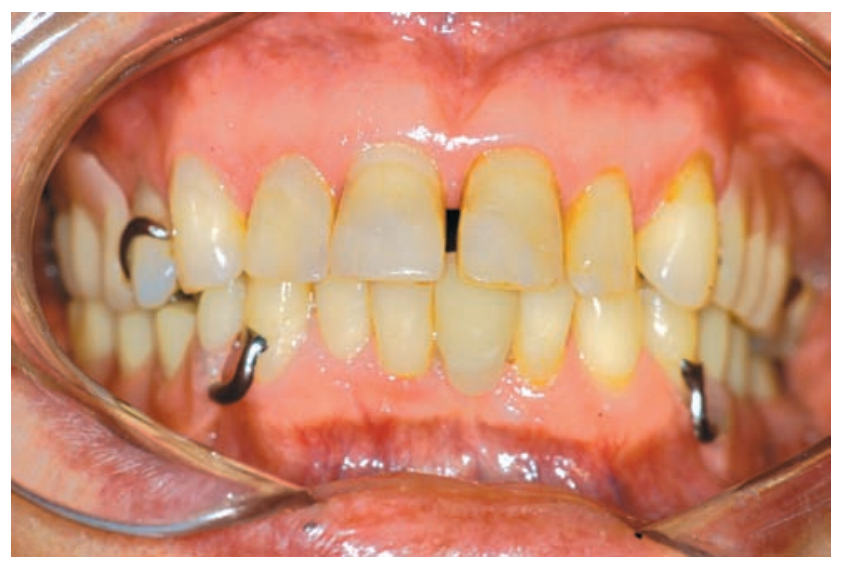

Fig. 12. Rehabilitación protésica y restauradora de la paciente de la figura 10. El material restaurados utilizado es ionómero de vidrio. 
GONZÁLEZ-ARRIAGADA, W. A.; SANTOS-SILVA, A. R.; CARVALHO DE ANDRADE M. A.; ELIAS, R. A. \& LOPES, M. A. Criterios de evaluación odontológica pre-radioterapia y necesidad de tratamiento de las enfermedades orales post-radioterapia en cabeza y cuello. Int. J. Odontostomat., 4(3):255-266, 2010.

Tabla III. Criterios de evaluación odontológica post-radioterapia

\begin{tabular}{|c|c|}
\hline Paciente desdentado & Paciente dentado parcial o total \\
\hline $\begin{array}{l}\text { 1. Confección de prótesis totales superior e inferior si es } \\
\text { posible }\end{array}$ & $\begin{array}{l}\text { 1. Confección de prótesis parciales si es necesario y/o } \\
\text { posible }\end{array}$ \\
\hline $\begin{array}{l}\text { 2. Orientaciones de higiene (uso de prótesis, limpieza con } \\
\text { gasa, colutorios) }\end{array}$ & $\begin{array}{l}\text { 2. Orientaciones de higiene (uso de cepillo e hilo dental, } \\
\text { colutorios con flúor, uso de prótesis) }\end{array}$ \\
\hline 3. Controles periódicos con protesista & $\begin{array}{l}\text { 3. Controles periódicos con dentista (cada tres meses } \\
\text { para tratamiento preventivo) }\end{array}$ \\
\hline 4. Evaluación y tratamiento de la & 4. Evaluación y tratamiento de la \\
\hline xerostomía/hiposalivación & xerostomía/hiposalivación \\
\hline $\begin{array}{l}\text { 5. Seguimiento profesional por recidivas y riesgo de } \\
\text { osteorradionecrosis }\end{array}$ & $\begin{array}{l}\text { 5. Seguimiento profesional por recidivas y riesgo de } \\
\text { osteorradionecrosis }\end{array}$ \\
\hline
\end{tabular}

Observación 1: en caso de ser necesario hacer exodoncias, el procedimiento se debe realizar bajo profilaxis antibiótica y manejando todas las herramientas de protección del coágulo. No se recomienda realizar más de dos exodoncias por sesión, siempre y cuando los dientes sean vecinos.

Observación 2: si es posible, es preferible optar por el tratamiento de endodoncia y luego amputación coronaria en lugar de la realización de una exodoncia.

Observación 3: en caso de enfermedad periodontal severa asintomática, se recomienda no realizar la exodoncia y esperar la exfoliación espontánea del diente involucrado.

deben mantener controles periódicos de su salud oral en general (Rosales et al.; Kielbasssa et al.; Papas et al.) (Tabla III). Han sido utilizadas técnicas con videos con buen resultado en los últimos años para educar a los pacientes (Krouse, 2000; Dunn et al.). Las complicaciones dentales post-radioterapia que se presentan pueden ocurrir debido a que los pacientes están más preocupados en cuidar de la enfermedad que en cuidar su propia salud bucal (Rosales et al.).

La osteorradionecrosis es la complicación postradioterápica más severa observada en pacientes sin evaluación odontológica pre-radioterapia (Rosales et al.). El tratamiento y control de la osteorradionecrosis son permanentes e imprevisibles (Lopes et al.; Németh et al.). Es aceptado que el riesgo de desarrollar osteorradionecrosis posterior a una extracción dental continua para toda la vida (Bonan et al., 2006).

Los controles periódicos por dentistas capacitados y un adecuado estado de la salud periodontal y oral en general durante y post-radioterapia, también son de vital importancia en el momento de hablar de prevención de osteorradionecrosis (Katsura et al.).
Aunque muchos dientes tengan indicación de exodoncia, normalmente ella es contraindicada en las áreas previamente irradiadas, por el riesgo de desarrollar osteorradionecrosis, por lo que el tratamiento endodóntico, dejando la raíz en el alvéolo, asume importancia, porque además de prevenir la osteorradionecrosis, posibilita el control de la sintomatología dolorosa y rehabilitación estética y funcional (Kielbassa et al.; Lopes et al.; Andrade et al.) (Figs. 11-12).

\section{CONCLUSIÓN}

La participación del cirujano-dentista en el equipo multidisciplinario responsable del tratamiento del paciente que será irradiado, aún más en la región de cabeza y cuello, es de vital importancia, a fin de minimizar las complicaciones durante y después de la radioterapia, mejorando la calidad de vida del paciente, siendo necesaria una evaluación odontológica previa de todo paciente antes de la radioterapia. 
GONZÁLEZ-ARRIAGADA, W. A.; SANTOS-SILVA, A. R.; CARVALHO DE ANDRADE M. A.; ELIAS, R. A. \& LOPES, M. A. Criterios de evaluación odontológica pre-radioterapia y necesidad de tratamiento de las enfermedades orales post-radioterapia en cabeza y cuello. Int. J. Odontostomat., 4(3):255-266, 2010.

GONZÁLEZ-ARRIAGADA, W. A.; SANTOS-SILVA, A. R.; CARVALHO DE ANDRADE M. A.; ELIAS, R. A. \& LOPES, M. A. Pre-radiotherapy dental evaluation criteria and treatment needs of oral side effects after head and neck radiotherapy. Int. J. Odontostomat., 4(3):255-266, 2010.

ABSTRACT: The objective of this review is to present pre-radiotherapy evaluation criteria and the main needs for treatment of these patients after the radiation therapy. Were revised articles in English, Spanish and Portuguese language between 1995 and 2009 indexed in Pubmed and Scielo. The keywords were oral cancer and radiotherapy, complications in head and neck radiotherapy, oral pre-radiotherapy evaluation. The adverse complications of radiotherapy in head and neck area could be temporary or late. The late effects, such as radiation caries and osteorradionecrosis could be directly associated with the fact that previous dental evaluation was not performed and can severely affect the post-operatory quality of life. The participation of the dentist in the multidisciplinary team and dental evaluation of the patients that will receive radiotherapy in the head and neck area are of vital importance to improve the post-operatory quality of life of these patients.

KEY WORDS: radiotherapy, head and neck cancer, adverse effects, treatment needs.

\section{REFERENCIAS BIBLIOGRÁFICAS}

Andrade, C. R.; Lopes, S. M. P.; Coletta, R. D.; Vargas, P. A. \& Lopes, M. A. Tratamento endodôntico em pacientes submetidos a radioterapia na região de cabeça e pescoço. Rev. Asocc. Paul. Cir. Dent., 57:43-6, 2003.

Andrews, N. \& Grifiths, C. Dental complications of head and neck radiotherapy. Part 1. Aust. Dent. J., 46:8894, 2001

Bonan, P. R. F.; Pires, F. R.; Lopes, M. A. \& Di Hipolito, O. Jr. Evaluation of salivary flow in patients during head and neck radiotherapy. Pesqui. Odontol. Bras., 17:15660, 2003.

Bonan, P. R. F.; Lopes, M. A.; Pires, F. R. \& Almeida, O. P. Dental management of low socioeconomic level patients before radiotherapy of the head and neck with special emphasis on the prevention of osteoradionecrosis. Braz. Dent. J., 17:336-42, 2006.

Caribé-Gomes, F.; Chimenos-Küstner, E.; López-López, J.; Finestres-Zubeldia, F. \& Guix-Melcior, B. Manejo odontológico de las complicaciones de la radioterapia y quimioterapia en el cáncer oral. Med. Oral, 8:17887, 2003.

Corvò, R.; Amichetti, M.; Ascarelli, A.; Arcangeli, G.; Buffoli, A.; Cellini, N.; Cionini, L.; De Renzis, C.; Emiliani, E.; Franchini, P.; Gabriele, P.; Gobitti, C.; Grillo Ruggieri, F.; Bertoni, F.; Magrini, S. M.; Marmiroli, L.; Orsatti, M.; Panizza, G. M.; Tordiglione, M.; Ziccarelli, L.; Gava, A.; Zorat, P. L.; Ghelfi, R.; Serra, G. F. \& Vitale, V. Effects of fluconazole in the prophylaxis of oropharyngeal candidiasis in patients undergoing radiotherapy for head and neck tumour: results from a double-blind placebo-controlled trial. Eur. J. Cancer Care, 17:270-7, 2008.
Chambers, M. S.; Garden, A. S.; Kies, M. S. \& Martin, J. W. Radiation-induced xerostomia in patients with head and neck cancer: pathogenesis, impact on quality of life, and management. Head Neck, 26:796-807, 2004.

Chitra, S. \& Shyamala Devi, C. S. Effects of radiation and a-tocopherol on saliva flow rate, amylase activity, total protein and electrolyte levels in oral cavity cancer. Indian J. Dent. Res., 19:213-8, 2008.

Chua, D. T. T.; Lo, C.; Yuen, J. \& Foo, Y. A pilot study of pentoxifylline in the treatment of radiation-induced trismus. Am. J. Clin. Oncol., 24:366-9, 2001.

Dirix, P.; Nuyts, S. \& Van den Boagert, W. Radiationinduced xerostomia in patients with head and neck cancer. Cancer; 107:2525-34, 2006.

Duncan, G. G.; Epstein, J. B.; Tu, D.; El Sayed, S.; Bezjak, A.; Ottaway, J.; Pater, J. \& National Cancer Institute of Canada Clinical Trials Group. Quality of life, mucositis, and xerostomia from radiotherapy for head and neck cancers: a report from the ncic ctg hn2 randomized trial of an antimicrobial lozenge to prevent mucositis. Head Neck, 27:421-8, 2005.

Doerr, T. D. \& Marunick, M. T. Timing of edentulation and extraction in the management of oral cavity and oropharyngeal malignancies. Head Neck, 19:426-30, 1997.

Dunn, J.; Steginga, S. K.; Rose, P.; Scott, J. \& Allison, R. Evaluating patient education materials about radiation therapy. Patient Educ. Couns., 52:325-32, 2004.

Epstein, J. B.; Emerton, S.; Lunn, R.; Le, N. \& Wong, F. L. Pretreatment assessment and dental management of 
GONZÁLEZ-ARRIAGADA, W. A.; SANTOS-SILVA, A. R.; CARVALHO DE ANDRADE M. A.; ELIAS, R. A. \& LOPES, M. A. Criterios de evaluación odontológica pre-radioterapia y necesidad de tratamiento de las enfermedades orales post-radioterapia en cabeza y cuello. Int. J. Odontostomat., 4(3):255-266, 2010.

patients with nasopharyngeal carcinoma. Oral Oncol., 35:33-9, 1999.

Epstein, J. B.; Gorsky, M.; Hancock, P.; Peters, N. \& Sherlock, C. H. The prevalence of herpes simplex virus shedding and infection in the oral cavity of seropositive patients undergoing head and neck radiation therapy. Oral Surg. Oral Med. Oral Pathol. Oral Radiol. Endod., 94:712-6, 2002.

Garg, A. K. \& Malo, M. Manifestations and treatment of xerostomia and associated oral effects secondary to head and neck radiation therapy. J. Am. Dent. Assoc., 128:1128-33, 1997.

Greenlee, R. T.; Murray, T.; Bolden, S. \& Wingo, P. A. Cancer Statistics, 2000. C. A. Cancer J. Clin., 50:733, 2000.

Grötz, K. A.; Wüstenberg, P.; Kohnen, R.; Al-Nawas, B.; Henneicke-Von Zepelin, H. H.; Bockisch, A.; Kutznerg, J.; Naser-Hijazih, B.; Belzi, G. G. \& Wagnerj, W. Prophylaxis of radiogenic sialadenitis and mucositis by coumarin/troxerutine in patients with head and neck cancer - a prospective, randomized, placebocontrolled, double-blind study. Br. J. Oral Maxillofac. Surg., 39:34-9, 2001.

Grötz, K. A.; Genitsariotis, S.; Vehling, D. \& Al-Nawas, B. Long-term oral Candida colonization, mucositis and salivary function after head and neck radiotherapy. Support Care Cancer; 11:717-21, 2003.

Guchelaar, H. J.; Vermes, A. \& Meerwaldt, J. H. Radiationinduced xerostomia: pathophysiology, clinical course and supportive treatment. Support Care Cancer; 5:281-8, 1997.

Hancock, P. J.; Epstein, J. B. \& Sadler, G. R. Oral and dental management related to radiation therapy for head and neck cancer. J. Can. Dent. Assoc., 69:58590, 2003.

Handschel, J.; Sunderkötter, C.; Kruse-Lösler, B.; Prott, F-J.; Meyer, U.; Piffko, J. \& Joos, U. Late effects of radiotherapy on oral mucosa in humans. Eur. J. Oral Sci., 109:95-102, 2001.

Herrstedt, J. Prevention and management of mucositis in patients with cancer. Int. J. Antimicrob. Agents, 16:1613, 2000.

Jensen, S. B.; Pedersen, A. M.; Reibel, J. \& Nauntofte, B. Xerostomia and hypofunction of the salivary glands in cancer therapy. Support Care Cancer, 11:207-25, 2003.
Jereczek-Fossa, B. \& Orecchia, R. Radiotherapyinduced mandibular bone complications. Cancer Treat. Rev., 28:65-74, 2002.

Jham, B. C. \& da Silva Freire, A. R. Oral complications of radiotherapy in the head and neck. Rev. Bras. Otorrinolaringol., 72:704-8, 2006.

Jham, B. C.; Reis, P. M.; Miranda, E. L.; Lopes, R. C.; Carvalho, A. L.; Scheper, M. A. \& Freire, A. R. Oral health status of 207 head and neck cancer patients before, during and after radiotherapy. Clin. Oral Invest., 12:19-24, 2008.

Katsura, K.; Sasai, K.; Sato, K.; Saito, M.; Hoshina, H. \& Hayashi, T. Relationship between oral health status and development of osteoradionecrosis of the mandible: A retrospective longitudinal study. Oral Surg. Oral Med. Oral Pathol. Oral Radiol. Endod., 105:731-8, 2008.

Katz, M. R.; Kopek, N.; Waldron, J.; Devins, G. M, \& Tomlinson, G. Screening for depression in head and neck cancer. Psychooncology, 13:269-80, 2004.

Keefe, D. M.; Schubert, M. M.; Elting, L. S.; Sonis, S. T.; Epstein, J. B.; Raber-Durlcher, J. E.; Migliorati, C. A.; McGuire, D. B.; Hutchins, R. D.; Peterson, D. E.; Mucositis Study Section of the Multinational Association of Supportive Care in Cancer and the International Society for Oral Oncology. Updated clinical practice guidelines for the prevention and treatment of mucositis. Cancer, 109:820-31, 2007.

Kielbassa, A. M.; Attin, T.; Schaller, H. G. \& Hellwig, E. Endodontic therapy in a postirradiated child: review of the literature and report of a case. Quintessence Int., 26:405-11, 1995.

Kielbassa, A. M.; Hinkelbein, W.; Hellwig, E. \& MeyerLückel, H. Radiation-related damage to dentition. Lancet Oncol., 7:326-35, 2006.

Koc, M. \& Aktas, E. Prophylactic treatment of mycotic mucositis in radiotherapy of patients with head and neck cancers. Jpn. J. Clin. Oncol., 3357-60, 2003.

Konings, A. W. T.; Coppes, R. P. \& Vissink, A. On the Mechanism of Salivary Gland Radiosensitivity. Int. J. Radiat. Oncol. Biol. Phys., 62:1187-94, 2005.

Kostler, W. J.; Hejna, M.; Wenzel, C. \& Zielinski, C. C. Oral mucositis complicating chemotherapy and/or radiotherapy: options for prevention and treatment. C. A. Cancer J. Clin., 51:290-315, 2001. 
GONZÁLEZ-ARRIAGADA, W. A.; SANTOS-SILVA, A. R.; CARVALHO DE ANDRADE M. A.; ELIAS, R. A. \& LOPES, M. A. Criterios de evaluación odontológica pre-radioterapia y necesidad de tratamiento de las enfermedades orales post-radioterapia en cabeza y cuello. Int. J. Odontostomat., 4(3):255-266, 2010.

Krouse, J. H. Video modelling to educate patients. J. Adv. Nurs., 33:748-57, 2001.

Lopes, M. A.; Coletta, R. D.; Alves, F. A.; Abbade, N. \& Rossi, J. R. A. Reconhecendo e controlando os efeitos colaterais da radioterapia. Rev. Asocc. Paul. Cir. Dent., 52:241-4, 1998.

Madan, P. D. K.; Sequeira, P. S.; Shenoy, K. \& Shetty, J. The effecte of three mouthwashes on radiationinduced mucositis in patients with head and neck malignancies: A randomized control trial. J. Cancer Res. Ther., 4:3-8, 2008.

Malouf, J. G.; Aragon, C.; Henson, B. S.; Eisbruch, A. \& Ship, J. A. Influence of parotid-sparing radiotherapy on xerostomia in head and neck cancer patients. Cancer Detect Prevent., 27:305-10, 2003.

Miller, E. H. \& Quinn, A. I. Dental considerations in the management of head and neck cancer patients. Otolaryngol. Clin. N. Am., 39:319-29, 2006.

Németh, Z.; Somogyi, A.; Takácsi-Nagy, Z.; Barabás, J.; Németh, G. \& Szabó, G. Possibilities of preventing osteoradionecrosis during complex therapy of tumors of the oral cavity. Pathol. Oncol. Res., 6:53-58, 2000.

Nicolatou-Galitis, O.; Sotiropoulou-Lontou, A.; Velegrakic, A.; Pissakass, G.; Kolitsi, G.; Kyprianou, K.; Kouloulias, V.; Papanikolaou, I.; Yiotakis, I. \& Dardoufas, K. Oral candidiasis in head and neck cancer patients receiving radiotherapy with amifostine cytoprotection. Oral Oncol., 39:397-401, 2003.

Niedermeier, W.; Matthaeus, C.; Meyer, C.; Staar, S.; Müller, R. P. \& Schulze, H. J. Radiation-induced hyposalivation and its treatment with oral pilocarpine. Oral Surg. Oral Med. Oral Pathal. Oral Radiol. Endod., 86:541-9, 1998.

Ochsenius, G.; Ormeño, A.; Godoy, L. \& Rojas, R. Estúdio retrospectivo de 232 casos de cáncer y precáncer de labio en pacientes chilenos: Correlación clínicohistológica. Rev. Méd. Chile, 131:60-6, 2003.

Oh, H. K.; Chambers, M. S.; Garden, A. S.; Wong, P. F. \& Martin, J. W. Risk of osteoradionecrosis after extraction of impacted third molars in irradiated head and neck cancer patients. J. Oral Maxillofac. Surg., 62:139-44, 2004.

Öhrn, K. E. O.; Whalin, Y-B. \& Sjödén, P-O. Oral status during radiotherapy and chemotherapy: a descriptive study of patient experiences and the occurrence of oral complications. Support Care Cancer, 9:247-257, 2001.
Papas, A.; Russel, D.; Singh, M.; Kent, R.; Triol, C. \& Winston, A. Caries clinical trial of a remineralising toothpaste in radiation patients. Gerodontology, 25:7688, 2008.

Pow, E. H. N.; McMillan, A. S.; Keung Leung, W.; Yong, M. C. M. \& Kwong, D. L. W. Salivary gland function and xerostomia in southern Chinese following radiotherapy for nasopharyngeal carcinoma Clin. Oral Invest., 7:230-4, 2003.

Pow, E. H. N.; Kwong, D. L. W.; McMillan, A. S.; Wong, M. C. M.; Sham, J. S. T.; Leung, L. H. T. \& Leung, W. K. Xerostomia and quality of life after intensity-modulated radiotherapy vs. conventional radiotherapy for earlystage nasopharyngeal carcinoma: initial report on a randomized controlled clinical trial. Int. J. Radiat. Oncol. Biol. Phys., 66:981-91, 2006.

Ramirez-Amador, V.; Silverman, S. Jr.; Mayer, P.; Tyler, M. \& Quivey. J. Candidal colonization and oral candidiasis in patients undergoing oral and pharyngeal radiation therapy. Oral Surg. Oral Med. Oral Pathol. Oral Radiol. Endod., 84:149-53, 1997.

Redding, S. W.; Zellars, R. C.; Kirkpatrick, W. R.; McAtee, R. K.; Cáceres, M. A.; Fothergill, A. W:, Lopez-Ribot, J. L.; Bailey, C. W.; Rinaldi, M. G. \& Patterson, T. F. Epidemiology of Oropharyngeal Candida Colonization and Infection in Patients Receiving Radiation for Head and Neck Cancer. J. Clin. Microb., 37:3896-900, 1999.

Reddy, S. P.; Leman, C. R.; Marks, J. E. \& Emami, B. Parotid-sparing irradiation for cancer of the oral cavity: maintenance of oral nutrition and body weight by preserving parotid function. Am. J. Clin. Oncol., 24:3416, 2001.

Reuther, T.; Schuster, T.; Mende, D. \& Kübler, A. Osteoradionecrosis of the jaws as a side effect of radiotherapy of head and neck tumour patients - A report of a 30 year retrospective review. Int. J. Oral Maxillofac. Surg., 32:289-95, 2003.

Rosales, A. C.; Esteves, S. C.; Jorge, J.; Almeida, O. P. \& Lopes, M. A. Dental needs in Brazilian patients subjected to head and neck radiotherapy. Braz. Dent. J., 20:74-7, 2009.

Rosenthal, D. I. \& Trotti, A. Strategies for managing radiation-induced mucositis in head and neck cancer. Semin. Radiat. Oncol., 19:29-34, 2009.

Rubira, C. M. F.; Devides, N. J.; Úbeda, L. T.; Bortolucci, A. G. Jr.; Lauris, J. R.; Rubira-Bullen, I. R. \& Damante, J. H. Evaluation of some oral postradiotherapy 
GONZÁLEZ-ARRIAGADA, W. A.; SANTOS-SILVA, A. R.; CARVALHO DE ANDRADE M. A.; ELIAS, R. A. \& LOPES, M. A. Criterios de evaluación odontológica pre-radioterapia y necesidad de tratamiento de las enfermedades orales post-radioterapia en cabeza y cuello. Int. J. Odontostomat., 4(3):255-266, 2010.

sequelae in patients treated for head and neck tumors. Braz. Oral Res., 21:272-7, 2007.

Russo, G.; Haddad, R.; Posner, M. \& Machtay, M. Radiation treatment breaks and ulcerative mucositis in head and neck cancer. Oncologist, 13:886-98, 2008.

Schiødt, M. \& Hermund, N. U. Management of oral disease prior to radiation therapy. Support Care Cancer, 10:403, 2002.

Sharp, H.; Morris, J. C.; Van Waes C.; Gius, D.; CooleyZgela, T. \& Singh, A. K. High incidence of oral dysesthesias on a trial of gefitinib, paclitaxel, and concurrent external beam radiation for locally advanced head and neck cancers. Am. J. Clin. Oncol., 31:557-60, 2008.

Silva, A. R. S.; Alves, F. A.; Antunes, A.; Goes, M. F. \& Lopes, M. A. Patterns of demineralization and dentin reactions in radiation-related caries. Caries Res., 43:43-9, 2009.

Shih, A.; Miaskowski, C.; Dodd, M. J.; Stotts, N. A. \& McPhail, L. Mechanisms for Radiation-induced Oral Mucositis and the Consequences. Cancer Nurs., 26:222-9, 2003.

Suarez-Cunqueiro, M. M.; Schramm, A.; Schoen, R.; Seoane-Leston, J.; Otero-Cepeda, X. L.; Bormann, K. H.; Kokemueller, H.; Metzger, M.; Diz-Dios, P. \& Gellrich, N. C. Speech and Swallowing Impairment After Treatment for Oral and Oropharyngeal Cancer. Arch. Otolaryngol. Head Neck Surg., 134:1299-304, 2008.

Sciubba, J. J. \& Goldenberg, D. Oral complications of radiotherapy. Lancet Oncol., 7:175-83, 2006.

Scully, C. \& Epstein, J. B. Oral health care for the cancer patient. Eur. J. Cancer B Oral Oncol., 32B:281-92, 1996.

Scully, C.; Epstein, J. \& Sonis, S. Oral Mucositis: A Challenging Complication of Radiotherapy, Chemotherapy, and Radiochemotherapy: Part 1, Pathogenesis and Prophylaxis of Mucositis. Head Neck, 25:1057-70, 2003.

Scully, C.; Epstein, J. \& Sonis, S. Oral mucositis: a challenging complication of radiotherapy, chemotherapy, and radiochemotherapy. Part 2: diagnosis and management of mucositis. Head Neck, 26:77-84. 2004.

Sonis, S. T. Oral mucositis in cancer therapy. J. Support. Oncol., 2:3-8, 2004.
Specht, L. Oral complications in the head and neck radiation patient: Introduction and scope of the problem. Support Care Cancer, 10:36-9, 2002.

Thorn, J.; Hansen, H. S.; Specht, L. \& Bastbolt, L. Osteoradionecrosis of the Jaws: Clinical Characteristics and Relation to the Field of Irradiation. J. Oral Maxillofac. Surg., 58:1088-93, 2000.

Toljanic, J. A.; Heshmati, R. H. \& Bedard, J. F. Dental follow-up compliance in a population of irradiated head and neck cancer patients. Oral Surg. Oral Med. Oral Pathol. Oral Radiol. Endod., 93:35-8, 2002.

Trotti, A.; Bellm, L. A.; Epstein, J. B.; Frame, D.; Fuchs, H. J.; Gwede, C. K.; Komaroff, E., Nalysnyk, L. \& Zilberberg, M. D. Mucositis incidence, severity and associated outcomes in patients with head and neck cancer receiving radiotherapy with or without chemotherapy: a systematic literature review. Radiother. Oncol., 66:253-62, 2003.

Vissink, A.; Burlage, F. R.; Spijkervet, F. K. L.; Jansma, J. \& Coppes, R. P. Prevention and treatment of the consequences of head and neck radiotherapy. Crit. Rev. Oral Biol. Med., 14:213-25, 2003.

Wong, J. K.; Wood, R. E. \& McLean, M. Conservative management of osteoradionecrosis. Oral Surg. Oral Med. Oral Pathol. Oral Radiol. Endod., 84:16-21, 1997.

Direccion para correspondencia:

Wilfredo Alejandro González-Arriagada

Área de Semiologia

Faculdade de Odontologia de Piracicaba-UNICAMP

Av. Limeira, 901

Bairro Areão

Piracicaba

CEP: $13.414-903$

São Paulo

BRAZIL

Fono: + $55192106-5266$

Fax: + $55192106-5218$

Cel: + $55198859-1980$

Email: wilgonzalez@fop.unicamp.br

Recibido : 11-10-2010

Aceptado: 03-12-2010 